\title{
Energy loss measurements of inclined muon bundles in the Cherenkov water detector
}

\author{
R.P. Kokoulin ${ }^{1, *}$, N.S. Barbashina ${ }^{1}$, A.G. Bogdanov ${ }^{1}$, S.S. Khokhlov ${ }^{1}$, V.A. Khomyakov ${ }^{1}$, V.V. Kindin ${ }^{1}$, \\ K.G. Kompaniets ${ }^{1}$, G. Mannocchi ${ }^{2}$, A.A. Petrukhin ${ }^{1}$, O. Saavedra ${ }^{3, \dagger}$, G. Trinchero ${ }^{2}$, V.V. Shutenko ${ }^{1}$, \\ I.I. Yashin ${ }^{1}$, and E.A. Yurina ${ }^{1}$ \\ ${ }^{1}$ National Research Nuclear University MEPhI (Moscow Engineering Physics Institute), 115409 Moscow, Russia \\ ${ }^{2}$ Osservatorio Astrofisico di Torino - INAF, 10025 Torino, Italy \\ ${ }^{3}$ Dipartimento di Fisica dell' Universita di Torino, 10125 Torino, Italy
}

\begin{abstract}
An experiment on the measurements of the energy deposit of inclined cosmic ray muon bundles is being conducted at the experimental complex NEVOD (MEPhI). The complex includes the Cherenkov water calorimeter with a volume of $2000 \mathrm{~m}^{3}$ and the coordinate-tracking detector DECOR with a total area of $70 \mathrm{~m}^{2}$. The DECOR data are used to determine the local muon densities in the bundle events and their arrival directions, while the energy deposits (and hence the average muon energy loss) are evaluated from the Cherenkov calorimeter response. Average energy loss carries information about the mean muon energy in the bundles. The detection of the bundles in a wide range of muon multiplicities and zenith angles gives the opportunity to explore the energy range of primary cosmic ray particles from about 10 to $1000 \mathrm{PeV}$ in the frame of a single experiment with a relatively small compact setup. Experimental results on the dependence of the muon bundle energy deposit on the zenith angle and the local muon density are presented and compared with expectations based on simulations of the EAS muon component with the CORSIKA code.
\end{abstract}

\section{Introduction}

In several cosmic ray experiments (e.g., $[1,2])$ an excess of muons compared to simulations in extensive air showers (EAS) at ultra-high energies of primary particles has been revealed. In particular, it was found that the measured intensity of multi-muon events cannot be explained in the frame of contemporary hadron interaction models even under the assumption of an extremely heavy mass composition (iron group nuclei) at primary energies around $10^{18} \mathrm{eV}$ [3].

A key to the solution of the problem of the inconsistent muon bundle abundance in EAS at ultra-high energies, often referred to as the "muon puzzle", may give investigations of the energy characteristics of the muon component of the EAS and their variations with primary energy [4]. A possible approach to such investigations is the measurement of the energy loss of muon bundles in the detector material. The specific energy loss of muons in the region above hundreds $\mathrm{GeV}$ almost linearly increases with muon energy $(-d E / d x \approx a+b E)$, and the appearance of an excessive flux of high-energy muons should change the dependence of the average muon bundle energy deposit on the energy of the primary particle. An experiment to investigate the energy deposit of inclined muon bundles was started at the experimental complex NEVOD in 2012. The complex includes the Cherenkov

\footnotetext{
* e-mail: rpkokoulin@mephi.ru

$\dagger$ deceased
}

water calorimeter NEVOD [5] and the coordinate-tracking detector DECOR [6] which ensures a reliable identification of multi-muon events at large zenith angles (down to the horizon). The first measurement results and the approaches to the analysis of the data were reported at the preceding Symposia [7, 8]. In this paper, updated results of the NEVOD-DECOR based on the data accumulated over the period from July 2013 to February 2018 are presented and compared with simulations performed on the basis of the CORSIKA code [9].

\section{Experimental setup and muon bundle data}

The Cherenkov water detector (CWD) NEVOD [5] has an inner volume of $9 \times 9 \times 26$ cubic meters and is equipped with a spatial lattice of quasi-spherical measuring modules (QSM). Each QSM includes six FEU-200 photomultipliers with flat $15 \mathrm{~cm}$ diameter cathodes directed along the axes of the orthogonal coordinate system. In total, the measuring system contains 91 QSMs (546 PMTs), arranged in vertical strings (3 or 4 QSMs in each) with distances $2.5 \mathrm{~m}$ along the main axis of the water tank and $2.0 \mathrm{~m}$ across it and over the depth. Electronic systems of the detector ensure measurements of the signals from each PMT in a wide dynamic range from 1 to $10^{5}$ photoelectrons.

The coordinate-tracking detector DECOR [6] was specially designed for the registration of multi-particle events 
at large zenith angles. It has a total area of about $70 \mathrm{~m}^{2}$ and includes eight supermodules (SMs) deployed in the galleries of the NEVOD building from three sides of the CWD water tank. The sensitive area of each supermodule is $8.4 \mathrm{~m}^{2}$. The supermodule consists of 8 vertical planes of plastic streamer tube chambers with resistive cathode coating and two-dimensional system of external readout strips. The spacing between neighboring planes in the SM is $6 \mathrm{~cm}$. The spatial and angular accuracy of the muon track reconstruction in the supermodule is better than $1 \mathrm{~cm}$ and $1^{\circ}$, respectively.

In spring 2013, a new water purification system of the CWD was launched into operation. With this new system, the Cherenkov light attenuation length increased several times and exceeded the transverse sizes of the water reservoir; as a result, the total number of detected photoelectrons from single muons and muon bundles in the CWD increased more than twice compared to earlier measurements.

As an experimental measure of the muon bundle energy deposit in the CWD we use the sum, $\Sigma$, of the signals detected in all PMTs of the water calorimeter (in units of photoelectrons, ph.e.). The local muon density of muons, $D\left(\mathrm{~m}^{-2}\right)$, in the event is estimated on the basis of the number of detected muons and effective area, $S_{\text {det }}$, of DECOR SMs for the measured direction of muon bundle arrival. The analysis showed that, to a first approximation, the total energy deposit in the CWD is proportional to the local muon density. Therefore we further consider the specific energy deposit $\Sigma / D$, that is, the Cherenkov calorimeter response normalized to the local muon density in the event.

The present analysis is based on the data accumulated over the period from July 2013 to February 2018. The total live time of observations amounted to 30,688 hours. From these data, 52,141 muon bundles with zenith angles greater than $55^{\circ}$ and with muon track multiplicities not less than 5 have been selected. More details concerning the procedure and the criteria of the selection of muon bundles in the DECOR data may be found elsewhere $[3,8]$.

\section{Results and discussion}

In Fig. 1, the zenith-angular dependence of the average specific energy deposit of muon bundles is presented. Experimental points are corrected for the angular-dependent contribution of the Cherenkov light reflected from the surface of the CWD water tank; this correction was calculated by means of the simulation of the passage of artificial muon bundles (with a fixed muon density and fixed energy) through the CWD in the frame of the Geant4 package $[10,11]$. The arrows in the figure indicate typical primary particle energies (calculated mean-logarithmic values [1]) which contribute to the formation of muon bundles at various zenith angles. The experimental data clearly demonstrate the increase of the mean energy of muons in the bundles with increasing zenith angle.

The curves in Fig. 1 represent the expected angular dependence of the specific energy deposit of muon bundles obtained on the basis of simulation of EAS by means of the CORSIKA code [9] for protons and iron nuclei as

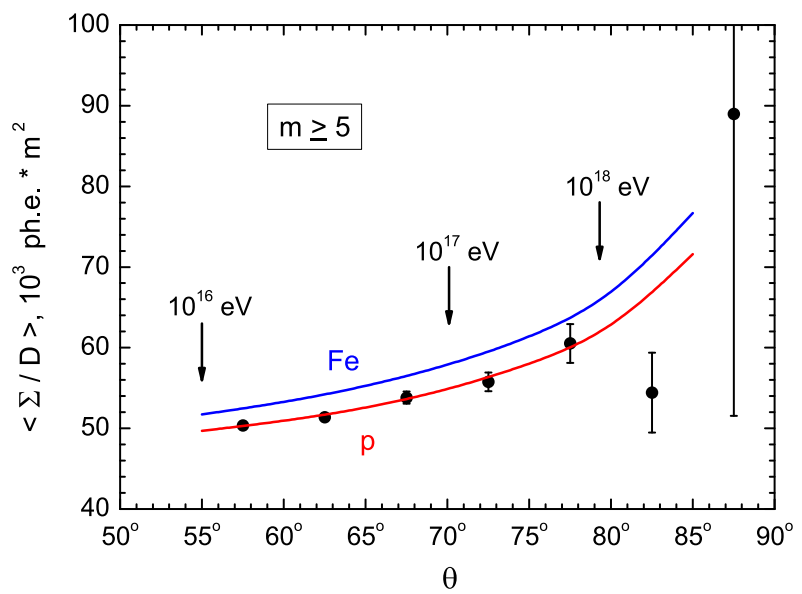

Figure 1. Dependence of the average specific energy deposit of muon bundles on the zenith angle. The points correspond to the present data. The curves represent results of CORSIKA-based calculations for primary protons and iron nuclei (labels ' $\mathrm{p}$ ' and 'Fe', respectively).

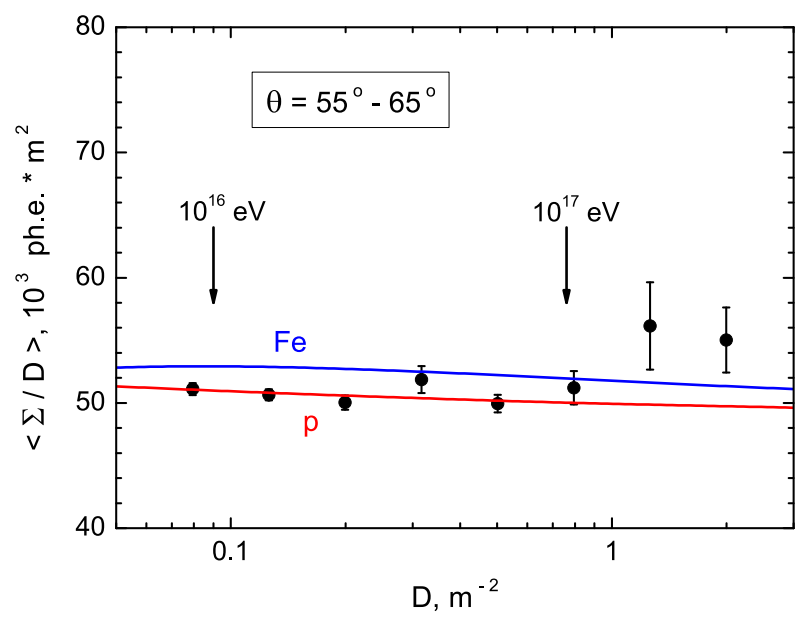

Figure 2. Dependence of the average specific energy deposit for muon bundles with arrival zenith angles $55^{\circ} \leq \theta<65^{\circ}$ on the local muon density. Notations are the same as in Fig. 1.

primary particles. In simulations, a combination of hadron interaction models SIBYLL2.3 [12] and FLUKA [13] for hadrons with energies above and below $80 \mathrm{GeV}$, respectively, was used. The calculated curve for primary protons was normalized to measurement results in the zenith angle interval $55^{\circ}-65^{\circ}$. As a whole, it can be seen from Fig. 1 that the measured dependence of the average specific energy deposit on the zenith angle is in good agreement with expectation.

In Fig. 2, the measured average specific energy deposit of muon bundles detected in the zenith angle interval $55^{\circ} \leq \theta<65^{\circ}$ is plotted as a function of the local muon density. It should be underlined that such a consideration for a fixed interval of zenith angles allows to search for possible changes of the detector response (and correspondingly, of the mean muon energy in the bundles) with the increase of the primary energy. The curves in the figure 


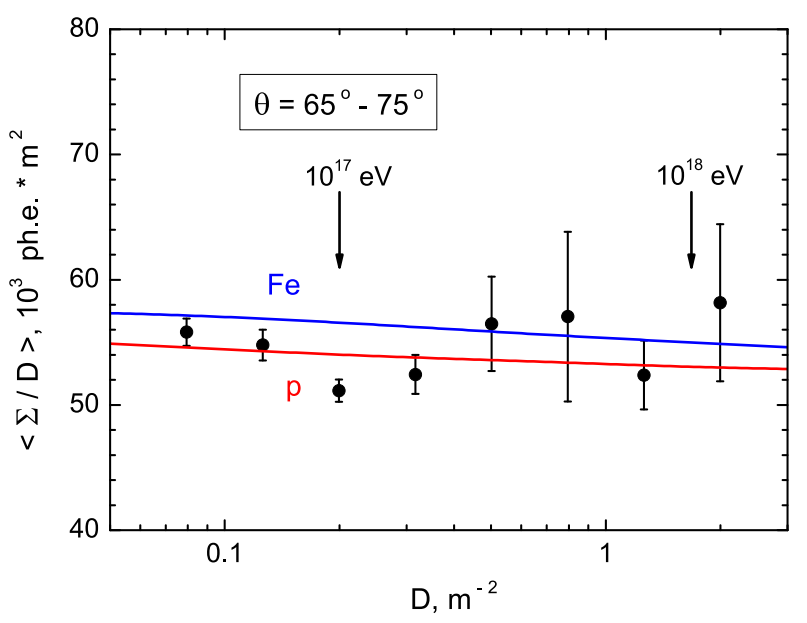

Figure 3. The same as in Fig. 2, but for muon bundles in the zenith angle interval $65^{\circ} \leq \theta<75^{\circ}$.

are obtained on the basis of simulation for primary protons and iron nuclei for a fixed zenith angle $\theta=59^{\circ}$; the same normalization coefficient as in Fig. 1 was applied. Simulation results demonstrate a trend to a slow decrease of the mean muon energy for increasing primary energy; on the contrary, the data give an indication for an increase of the specific energy deposit at primary energies exceeding $10^{17} \mathrm{eV}$. In Fig. 3, the same dependence as in Fig. 2 but for zenith angle interval $65^{\circ} \leq \theta<75^{\circ}$ is presented. Noticeably, the shift in zenith angle interval being considered leads to the increase of effective primary energies corresponding to the same local muon density in the bundles. Though statistical errors for the moment are large, evidence for an increase of the average specific energy deposit of muon bundles between primary energies $10^{17}$ and $10^{18} \mathrm{eV}$ is also seen.

\section{Conclusion}

An experiment on the measurements of the energy loss of muon bundles in inclined EAS is being conducted at the NEVOD-DECOR complex. For the moment, preliminary analysis of data accumulated for about 30.7 thousand hours of observations has been performed. An appreciable dependence of the average specific energy deposit (normalized to the muon density) on the zenith angle has been revealed. This dependence is in a good agreement with CORSIKA-based simulations. An indication for an increase of the mean muon energy in the bundles in comparison with expectation at primary energies between $10^{17}$ and $10^{18} \mathrm{eV}$ has been found. This deviation, if confirmed, may be evidence for an inclusion of a new mechanism of highenergy muon generation at these primary energies. However, a further increase of statistics and a careful analysis of possible systematic uncertainties are necessary. Accumulation of experimental data, their processing, simulations and data analysis are being continued.

\section{Acknowledgements}

The work has been performed at the Unique Scientific Facility "Experimental Complex NEVOD" with a state support from the Ministry of Science and Higher Education of the Russian Federation (MEPhI Academic Excellence Project and government task) and the Russian Foundation for Basic Research (grant no. 18-02-00971-a). Simulations were performed using the resources of the MEPhI high-performance computing center.

\section{References}

[1] A.G. Bogdanov et al., Phys. Atom. Nuclei 73, 1852 (2010)

[2] A. Aab et al., Phys. Rev. D 91, 032003 (2015)

[3] A.G. Bogdanov et al., Astropart. Phys. 98, 13 (2018)

[4] A.A. Petrukhin, NIM A 742, 228 (2014)

[5] A.A. Petrukhin, Physics-Uspekhi 58, 486 (2015)

[6] N.S. Barbashina et al., Instrum. Experim. Techniques 43, 743 (2000)

[7] A.G. Bogdanov et al., EPJ Web of Conf. 99, 06004 (2015)

[8] R.P. Kokoulin et al., EPJ Web of Conf. 145, 16002 (2017)

[9] D. Heck et al., Forschungszentrum Karlsruhe Report, FZKA 6019 (1998)

[10] S. Agostinelli et al., NIM A 506, 250 (2003)

[11] J. Allison et al., NIM A 835, 186 (2016)

[12] F. Riehn et al., Proc. 34th Int. Cosmic Ray Conf., Hague, PoS (ICRC2015) 558 (2015)

[13] A. Ferrari et al., Report CERN-2005-10 (2005) 